\title{
Design, Synthesis and Evaluation of Heterogeneous FPGA with Mixed LUTs and Macro-Gates .
}

\author{
Yu Hu, ${ }^{1,2}$, Satyaki Das ${ }^{2}$, Steve Trimberger ${ }^{2}$ and Lei $\mathrm{He}^{1}$ \\ Electrical Engineering Department, UCLA, Los Angeles, CA $90095^{1}$ \\ Research Laboratory, Xilinx Inc., 2100 Logic Dr. San Jose, CA 95124²
}

\begin{abstract}
Small gates, such as AND2, XOR2 and MUX2, have been mixed with lookup tables (LUTs) inside the programmable logic block (PLB) to reduce area and power and increase performance in FPGAs. However, it is unclear whether incorporating macro-gates with wide inputs inside PLBs is beneficial. In this paper, we first propose a methodology to extract a small set of logic functions that are able to implement a large portion of functions for given FPGA applications. Assuming that the extracted logic functions are implemented by macro-gates in PLBs, we then develop a complete synthesis flow for such heterogeneous PLBs with mixed LUTs and macro-gates. The flow includes a cut-based delay and area optimized technology mapping, a mixed binary integer and linear programming based area recovery algorithm to balance the resource utilization of macro-gates and LUTs for area-efficient packing, and a SAT-based packing. We finally evaluate the proposed heterogeneous FPGA using the newly developed flow and show that mixing LUT and macro-gates, both with 6 inputs, improves performance by $16.5 \%$ and reduces logic area by $30 \%$ compared to using merely 6-input LUTs.
\end{abstract}

\section{INTRODUCTION}

The popular island style FPGA architecture [1] consists of programmable logic blocks (PLBs) embedded in routing channels. The logic element within the PLB can be a lookup table (LUT) [2], programmable logic array (PLA) [3], or macro-gate (e.g. AND gates and multiplexers) [4]. These logic elements offer a spectrum of trade-offs between functionality and costs in terms of area, power and delay. For instance, a circuit can be implemented by fewer $K$-input LUTs than $K$-input macro-gates, while a $K$-input macro-gate requires smaller silicon area and has lower propagation delay than a $K$-input LUT. The PLB is heterogeneous if it consists of different types of logic elements, otherwise it is homogeneous. In this paper, we assume that heterogeneity exists only inside a PLB while the structures of all PLBs are identical, and study the impact of heterogeneous PLBs.

Recent work has shown when uniform LUTs are used for the PLB, a larger LUT size increases performance [5, 6], but it reduces the LUT pin utilization rate. For example, mapping IWLS'05 benchmarks [7] using 6-input LUTs and the Berkeley ABC mapper [8], we find that over $60 \%$ of the LUTs use less than five inputs. Initial studies in the literature have suggested that mixed-sized LUTs [5, 9, 10, 26], mixing LUTs and PLAs [11] inside the PLB, or PLBs with hard-wired connections [27] may improve logic density. In addition, commercial FPGAs [5] have benefited from small macro-

\footnotetext{
* This paper is partially supported by NSF grant CCR-0306682. Address comments to lhe@ee.ucla.edu.
}

gates (e.g., XOR2 and MUX2) inside the PLB. However it is unclear whether incorporating macro-gates with wide inputs inside PLB is beneficial. The first contribution of this paper is to propose a methodology to extract a small set of logic functions that are able to implement a large portion of functions for given FPGA applications. Assuming that the extracted logic functions are implemented by macro-gates in PLB, we design a heterogeneous PLB consisting of both LUTs and macro-gates.

Effective and efficient synthesis tools are key enablers for the exploration of different architecture options. There are extensive studies (e.g., [12, 13, 14, 15, 16, 17]) on synthesis for homogeneous PLBs, but only limited research on synthesis for heterogeneous PLBs. [10] proposed heuristics to speedup the technology mapping for homogeneous PLBs and then extended them to consider heterogeneous PLBs with mixed LUT sizes. [18, 19] integrated Boolean Satisfiability solvers into re-synthesis to deal with macrogates in heterogeneous PLBs. However, the high time complexity prohibits exploring complicated heterogeneous PLBs. The second contribution of this work is to develop an efficient logic synthesis flow for heterogeneous PLBs. The flow includes a cut-based delayoptimal technology mapping, a mixed binary integer and linear programming (MBILP) based area recovery algorithm to balance the resource utilization of macro-gates and LUTs for area-efficient packing, and a SAT-based packing. Using the newly developed flow, we evaluate the proposed heterogeneous PLB, and show that mixing LUT and macro-gates, both with 6 inputs, improves performance by $16.5 \%$ and reduces logic area by $30 \%$ for IWLS' 05 benchmarks [7] when compared to using 6-input LUTs.

The rest of this paper is organized as follows. Section 2 presents the methodology to design heterogeneous PLBs with mixed LUTs and macro-gates. Section 3 describes the improved technology mapping and post-mapping area recovery algorithms to deal with the proposed FPGA architecture. Section 4 proposes a flexible SAT-based packing algorithm. The architecture evaluation results are given in Section 5, and the paper is concluded in Section 6. To the best of our knowledge, this paper is the first systematic study of the logic synthesis flow for FPGAs consisting of heterogeneous PLBs with wide-input macro-gates.

\section{HETEROGENEOUS PLB DESIGN}

The key step in designing a heterogeneous PLB is to extract a small set of logic functions that are able to implement a large portion of functions in given FPGA applications. In this section, we discuss how to extract such a set of logic functions by performing logic function ranking, and then present our macro-gate design.

\subsection{Preliminaries}

To rank the logic functions extracted from the training FPGA 
application set, we need to identify two important relationships i.e., $\mathrm{NPN}$-equivalence and inheritance equivalence, for every two logic functions.

DEFINITION 1. (NPN Equivalence) Two boolean functions, $F$ and $G$, belong to the same $N P N$-class (NPN-equivalent) if $F$ can be derived from $G$ by negating ( $N$ ) and permuting $(P)$ inputs and negating $(N)$ the output. [20]

For example, $F=a b+c$ and $G=a^{\prime} c+b$ are NPN-equivalent. Although there are $2^{2^{4}}=65536$ different 4-variable functions, only 222 of them are NPN independent (i.e., NPN-inequivalent to each other). In the rest of this paper, we use the term "NPN-class" to denote a set containing only NPN-independent elements. In this work, we limit the programmability of a macro-gate with input/output negation, which provides a good trade-off between flexibility and cost (performance and area). For an $N$-input macro-gate with input/output negation, $N+1$ memory cells (i.e., configuration bits) are needed rather than $2^{N}$ memory cells for a LUT with $N$ inputs $($ LUT- $N)$.

DEFINITION 2. (Inheritance Equivalence) For logic function $A=F_{1}\left(a_{1}, \cdots, a_{n}\right)$ and $B=F_{2}\left(b_{1}, \cdots, b_{m}\right)$, where $0 \leq m<n, A$ is inheritance equivalent to $B$ iff $\exists a_{i}$ s.t. $A\left(a_{1}, \cdots, a_{i}=1, \cdots, a_{n}\right)$ or $A\left(a_{1}, \cdots, a_{i}=0, \cdots, a_{n}\right)$ and $B$ are $N P N$-equivalent.

In the other words, $A$ and $B$ are NPN-equivalent if we fix one of the inputs of $A$ as logic zero or one, i.e., $B$ is a cofactor of $A$. For example, $f_{1}(a, b, c, d)=a b c d$ is inheritance equivalent to $f_{2}(a, b, c)=a b c$ by fixing input $d$ as one. In fact, $f_{1}$ is a 4-input AND gate, which can be used to implement a 3-input AND gate (i.e. $f_{2}$ ). The inheritance equivalence of the full set of NPN-class with up to $N$ inputs can be represented by the following NPN-class diagram (NCD).

\subsection{Utilization NPN-Class Diagram (UND)}

To graphically organize the logic functions and represent their NPN Equivalence and Inheritance Equivalence relationships, we propose the following NPN-Class Diagram (NCD).

Definition 3. NPN-Class Diagram (NCD) is a directed acyclic graph (DAG) where each node in level $N$ is the representative ${ }^{1}$ of an $N$-input $N P N$-Class, and each edge from node $A$ to node $B$ indicates that function $A$ is inheritance equivalent to $B$, i.e. A can implement $B$ 's functionality.

Note that Inheritance Equivalence is asymmetric, e.g., $f_{1}$ is inheritance equivalent to $f_{2}$ but the reverse is not true. Therefore, NCD is a DAG as no edge is from lower level node to high level node. Figure 1 shows the NCD for all 3-input functions.

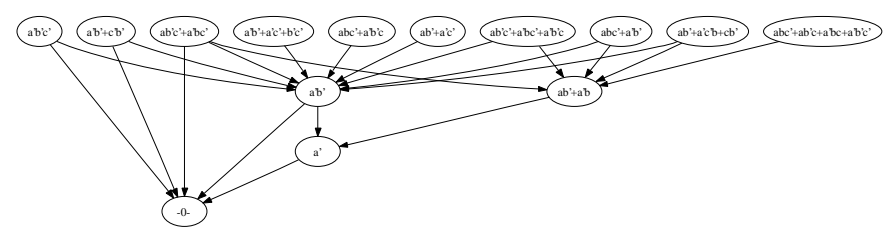

Figure 1: NCD for 3-input logic functions

\footnotetext{
${ }^{1}$ The representative (canonical form) of a $N$-input NPN-Class can be selected based on different rules, but the NCD is always applicable.
}

To extract $N$-input macro-gate logic functions from the training FPGA application set, we first map those applications by exclusive LUT- $N$ architecture and then analyze all logic functions that are mapped into LUTs. Note that NCD stores all different function categories and their relationships within a DAG, which makes it extremely efficient to explore some interesting properties of an application. To represent the functions implemented by each particular application based on NCD, we present the Utilization NPN-Class Diagram (UND), which is a sub-graph of NCD in addition to the weights associated with each node. UND can be defined recursively as follows.

DEFINITION 4. (Utilization NPN-Class Diagram (UND)) For a particular application D, utilization NPN-Class diagram $\mathcal{U}_{D}$ of $D$ is a sub-graph of NCD. If a function $F$ is implemented by at least one of the LUTs in D, the NCD node that corresponds to the NPN-Class of F should be added into $\mathcal{U}_{D}$. If a $N C D$ node is present in $\mathcal{U}_{D}$, all of its fanout nodes and edges should be added into $\mathcal{U}_{D}$ recursively. Each node is associated with a 3-tuple $\Phi(f, n, c)$, where $f$ is the functionality description of this NPN-Class, $n$ and $c$ are the implemented frequency and implementation capability (will be defined later), respectively.

DEFINITION 5. (Implemented Frequency (IF)) For a $N P N$-Class function $f$ presented in a mapped application, the implemented frequency $I F_{f}$ of logic function $f$ is the number of LUTs which implement logic function $f$.

DEFINITION 6. (Implementation Capability (IC)) For a NPN-Class function $f$ presented in the UND of a mapped application, the implementation capability $I C_{f}$ of $f$ is calculated by the following equation:

$$
I C_{f}=\frac{\sum_{\forall v \in \text { fanout cone of } f} I F_{v}}{\sum_{\forall u \in U N D} I F_{u}}
$$

Intuitively, $I C_{f}$ of NPN-Class function $f$ indicates the portion (in terms of percentage) of logic functions in the application that can be implemented by $f$.

Figure 2 shows the UND for a small application that is mapped by LUT-3. There are totally 12040 functions implemented by LUTs in this application, and only three 3-input NPN-classes are presented. An interesting observation from Figure 2 is that the IC for function $g=a b^{\prime} c+a^{\prime} b c^{\prime}+a^{\prime} b^{\prime} c$ is $55 \%$ while its IF is only 1120 (less than $10 \%$ of 12040 total functions). In fact, it has a child node $a b^{\prime}+a^{\prime} b$ whose IF is 4410 (36\% of 12040 total functions), if we look into the fanout cone (shadowed area) of node $a b^{\prime} c+a^{\prime} b c^{\prime}+a^{\prime} b^{\prime} c$.

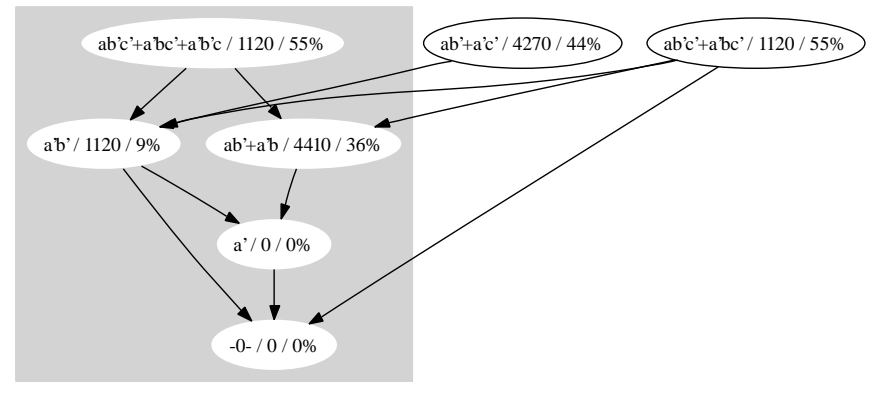

Figure 2: An example UND

As we are interested in the minimal number of logic gates to cover all logic functions in an application, i.e., minimal functional 
covering (MFC), we propose the following theorem to calculate the MFC very efficiently.

THEOREM 1. The number of minimal functional covering (MFC) in application $D$ is equal to the number of all primary input nodes (with no parent nodes) of UND for D.

The proof of this theorem is straightforward.

\subsection{UND for Wide Input Functions}

To explore functions with fewer than 5 inputs, we can build 4input NCD once and use a lookup table to store the NPN-class of every logic function. For each of the training applications that are mapped by LUT-4, we can find its NPN-class from the lookup table and create or label the node in UND. However, this procedure becomes prohibitively expensive since we cannot afford to preconstruct NCD for more than 4-input functions by exhaustively examining NPN-equivalence for even all 5-input functions $\left(2^{2^{5}}=\right.$ 4294967296).

Practically, one can build a partial $U N D$ by online checking NPN-equivalence for over-5-input functions, which can be performed efficiently by the method proposed in [20]. When a new node representing a $N$-input $(N \geq 5) \mathrm{NPN}$-class function is inserted in the partial UND, only those $(N-1)$-input functions that are inheritance equivalent to it are inserted/labeled in the partial UND, instead of performing insertion recursively. Partial UND is a good approximation of UND and all methods for UND manipulation are applicable for partial UND. In experiments, we find that the total number of all 6-input NPN-classes presented in all IWLS' 05 benchmarks [7] is less than 5000 and only 167 of them are present more than $1 \%$ out of all functions. Therefore, the size of partial UND can be well controlled in practice.

\subsection{Macro-Gate Design}

The logic function extraction and ranking framework proposed above is implemented by the mix of Perl and $\mathrm{C}$ on the Berkeley ABC [8] platform. Using IWLS'05 benchmarks [7] as the training set, the following 6-input NPN-classes with the highest ranks are found as the candidates of the macro-gates to be added into the FPGA PLB.

$$
\begin{aligned}
& g_{1}(a, b, c, d, e, f)=a b c d e f \\
& g_{2}(a, b, c, d, e, f)=a b^{\prime} c^{\prime}+b c f+b c^{\prime} d+b^{\prime} c e \\
& g_{3}(a, b, c, d, e, f)=a b^{\prime} c d^{\prime} e+b c e f+d e f \\
& g_{4}(a, b, c, d, e, f)=a b^{\prime}+a^{\prime} c d^{\prime}+b^{\prime} c^{\prime}+e^{\prime}+f^{\prime}
\end{aligned}
$$

Combining these four gates with input/output negation, we can implement 23\% 6-input functions, 34\% 5-input functions, 60\% 4input functions, 69\% 3-input functions and 98\% 2-input functions on average for IWLS'05 benchmarks. Overall $50 \%$ functions can be implemented by this macro-gate. The structure of the macrogate is shown in Figure 3. In our experiments, we incorporate this macro-gate into the FPGA PLBs and assume that there exist one LUT and one such macro-gate in each PLB.

\section{TECHNOLOGY MAPPING}

Given the functions of the macro-gates to be built into FPGA PLBs, a technology mapper is needed to make full use of these macro-gates in terms of performance improvement and area reduction. [18] presented a general algorithm for heterogeneous FPGA technology mapping, but it is not scalable to large macro-gates. [17] extended the traditional cut-based technology mapping algorithm

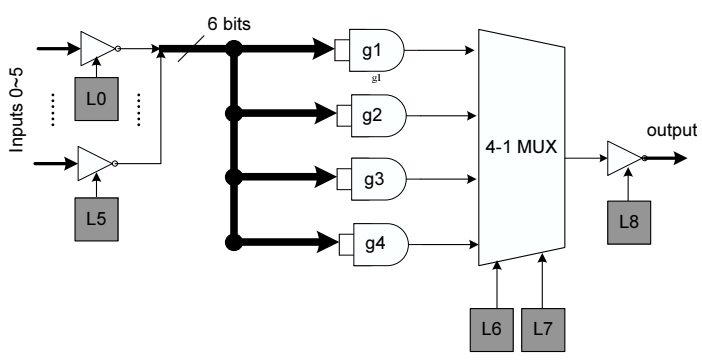

Figure 3: Architecture of the macro-gate

[10] (for LUT-only FPGAs) to achieve delay optimality for macrogate based FPGAs ${ }^{2}$ and proposed a novel concept, i.e., factor cuts, to improve the scalability of the algorithm. In [17], the truth table of the NPN-classes that can be implemented by the macro-gates (with $M$ inputs) are pre-calculated and stored in a lookup table. Then the mapping is done in two passes. The first pass is a forward topology traversal for enumerating all $M$-feasible cuts and keeping only those that can be found in the lookup table storing the truth tables of macro-gates. The second pass is a backward topology traversal to select the best cut for every node under the delay target and area constraints. For technology mapping to heterogeneous PLBs with both LUTs and macro-gates, we adopt the same framework presented in [17] by labeling cuts as macro-gates or LUTs based on their logic functions.

After the cut-based mapping, an area recovery phase is needed for the proposed macro-gate based heterogeneous FPGA architecture. For the target PLB with $C_{L}$ LUTs and $C_{M}$ macro-gates, suppose there are $N_{L}$ LUTs and $N_{M}$ macro-gates in the mapped circuit, the logic area (i.e., the number of PLBs) after the ideal packing should be

$$
\begin{aligned}
\Phi(N, \alpha) & =\max \left(\frac{N_{M}}{C_{M}}, \frac{N_{L}}{C_{L}}\right) \\
& =\frac{N}{C_{L}} \cdot \max \left(\frac{\alpha}{\beta}, 1-\alpha\right)
\end{aligned}
$$

where $N=N_{M}+N_{L}, \beta=C_{M} / C_{L}$ and $\alpha=N_{M} / N$.

Since $C_{L}$ and $\beta$ are constant for a given architecture, it is easy to show

$$
\begin{array}{r}
\min _{\forall \alpha} \Phi(N, \alpha)=\frac{N}{C_{L}} \cdot \frac{1}{1+\beta} \\
\alpha^{*}=\frac{\beta}{1+\beta}
\end{array}
$$

For a given architecture, (6) provides a lower bound of the number of PLBs for a mapped result, where parameters $N$ and $\alpha$ decide the tightness of the packing. (7) shows that the tightest packing can be achieved when the LUT-MG $\operatorname{ratios}^{3}$ of the given architecture $\left(\frac{\beta}{1+\beta}\right)$ and the mapped result $(\alpha)$ are equal.

For example, suppose the target architecture has one LUT and one macro-gate within a PLB, i.e., the LUT-MG ratio is $1: 1$. If we can achieve the same ratio in the mapped result, a tight packing is expected. In fact, we can adjust the area weight assigned to LUTs and macro-gates in the technology mapper, which shows significant impact in the LUT-MG ratio of the mapped result. Figure 4 shows the average number of LUTs and macro-gates in the mapped results for 20 IWLS' 05 benchmarks with different area weight assignments for a 6-input LUT and a 6-input macro-gate, i.e., 1:1, 1:0.95, 1:0.9,

\footnotetext{
${ }^{2}$ The macro-gate in [17] was built by three LUTs (without the logic gates) and the homogeneous PLBs were assumed.

${ }^{3}$ LUT-MG ratio is the number of LUTs divided by the number of macro-gates in an application.
} 
1:0.8, 1:0.5 and 1:0.1. The LUT-MG ratio can be clearly seen in this figure. To achieve a tight packing for the target architecture with LUT-MG ratio 1:1, we first perform a binary search to find the best area weight assignment which gives small $N$ and $\alpha$ close to the target LUT-MG ratio. From Figure 4, we see that the total number of LUTs and macro-gates $(N$ in (6)) increases dramatically if an extremely small weight is assigned to macro-gates (see the bar for 1:0.5 and 1:0.1) since the mapper is biased. We find that 1:0.95, 1:0.9 and 1:0.8 all give good trade-offs between $N$ and $\alpha$ under the target LUT-MG ratio but it is hard to further improve the resulting LUT-MG ratio, $\beta$, by simply adjusting area weight.

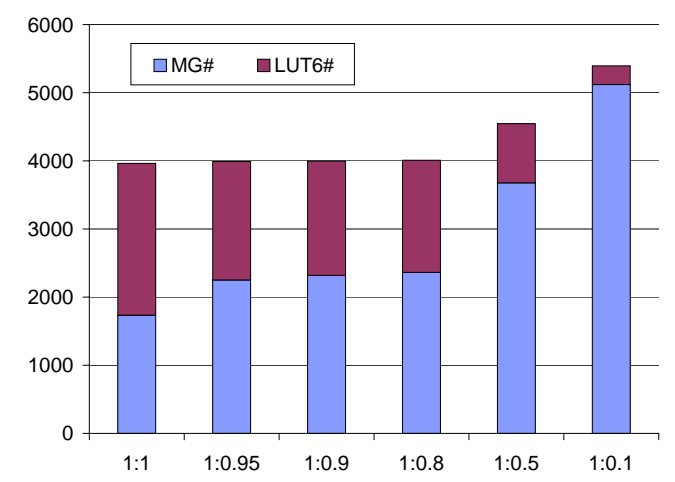

Figure 4: Impact of area weight on LUT-MG ratio

Given the mapped result after binary search, the total number of LUTs and macro-gates, $N$, and delay target, $T$, are fixed. Motivated by the timing slack budgeting [21], we can reassign macro-gates and LUTs in such a way that the resulting LUT-MG ratio is sufficiently close to the target LUT-MG ratio while preserving the delay target. The combinational portion of the mapped result is represented in a DAG $G=(V, E)$, where $\forall v_{j} \in V$ is mapped to a LUT or a macro-gate. Without loss of generality, we assume that the intrinsic delay of a LUT is $\Delta D$ larger than a macro-gate. In this case, the binary search should be able to find a LUT-MG ratio, $\alpha$, which is slightly smaller than the target LUT-MG ratio, $\beta /(1+\beta)$. To balance the number of LUTs and macro-gates according to $\beta$, certain macro-gates should be re-mapped as LUTs. For each node $v_{j}$ that is mapped as a macro-gate, if its input number is not larger than the LUT size, it can be re-mapped as a LUT without changing the functionality. All such nodes are stored in set $V_{m}$. A binary variable $m_{j}$ indicates if node $v_{j}$ can be re-mapped as a LUT for the given timing slack $b_{j}$. The objective is to minimize the gap between the mapped macro-gate number $\sum_{\forall v_{j} \in V_{m}(G)} m_{j}$ and the ideal number $\frac{1}{1+\beta} \cdot N$. The overall problem can be formulated as a mixed binary integer and linear programming (MBILP) as follows.

$$
\begin{array}{cc}
\min & \left|\sum_{\forall v_{j} \in V_{m}(G)} m_{j}-\frac{1}{1+\beta} \cdot N\right| \\
\text { s.t. } & m_{j} \leq \frac{b_{j}}{\Delta D}, \forall v_{i} \in V_{m}(G) \\
& m_{j} \in\{0,1\}, \forall v_{i} \in V_{m}(G) \\
a_{i}+d_{j}+b_{j} \leq a_{j}, \forall e(i, j) \in E(G) \\
b_{i} \geq 0, \forall v_{i} \in V(G) \\
a_{i} \leq T, \forall v_{i} \in V(G)
\end{array}
$$

where $a_{i}$ and $d_{j}$ are the arrival time and intrinsic delay for node $v_{i}$, respectively. All the other variables have been explained above. Note that the absolute operator in the objective function can be easily transformed into linear form by introducing an auxiliary variable $t$ as follows.

$$
\begin{array}{cc}
\min & t \\
\text { s.t. } & \sum_{\forall v_{j} \in V_{m}(G)} m_{j}-\frac{1}{1+\beta} \cdot N \leq t \\
& \sum_{\forall v_{j} \in V_{m}(G)} m_{j}-\frac{1}{1+\beta} \cdot N \geq-t \\
& t \geq 0
\end{array}
$$

\section{SAT BASED FLEXIBLE PLB PACKER}

Given the mapped application, the following step is to pack the logic elements, such as LUTs, FFs and macro-gates, into FPGA PLBs. The typical existing packing tools such as T-VPack [1] hard-codes the architecture specification of a PLB, a step requiring rewriting from scratch if the PLB architecture changes. This is time consuming and needs to be debugged carefully to avoid bugs. The flow proposed in [18] and [19] tries to solve technology mapping and packing simultaneously. It is extremely flexible but impractical for typical applications due to its high computational cost.

In contrast to the existing work, we solve the problem of validating PLB packing as a local place and route problem at the PLB level. This can be considered a placement problem since multiple compatible sites may be present for each logic element in the clus$\operatorname{ter}^{4}$. Also, for the chosen placement, all interconnections within the cluster must be routed in the PLB and all external connections must be connected to appropriate PLB input/output pins. We use a satisfiability (SAT) solver to carry out this PLB packing validation. The tool takes a netlist description of the target PLB, the user netlist, and the netlist elements clustered into the PLB to generate a set of Boolean equations such that each solution to these equations represents a valid packing. Then a SAT-solver can be used to find a solution to this set of equations. If a solution exists, the PLB packing is valid and an assignment of the netlist elements to locations in the PLB and PLB input or output pins to nets is produced. If no solution exists, the packing is invalid.

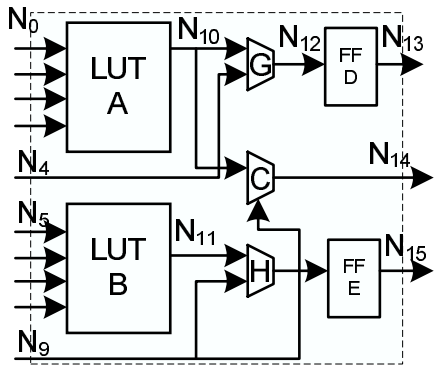

(a)

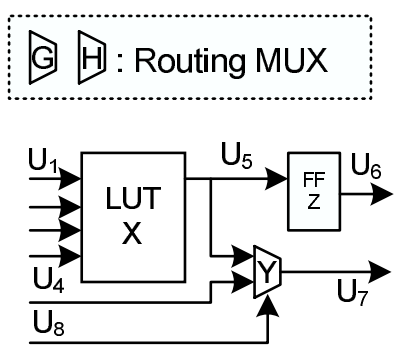

(b)
Figure 5: (a) Architecture of a FPGA PLB, (b) Subcircuit to be packed

Now we briefly sketch the problem formulation with an example. Assume that we are attempting to fit the fragment described in Figure 5 (b) into the simplified Xilinx Spartan3-like PLB in Figure 5 (a). We have variables $X @ A, X @ B$ encoding placement of LUT $X$ at either the site $A$ or $B$. Now we generate the exclusivity constraint and presence constraint as follows.

$$
\begin{array}{r}
(\neg X @ A) \vee(\neg X @ B) \\
X @ A \vee X @ B
\end{array}
$$

Constraint (16) implies that if $X$ is placed at $A$ then it cannot be placed at $B$ and vice versa. Constraint (17) means that $X$ must be

\footnotetext{
${ }^{4}$ For instance in Xilinx Spartan3 [4] PLB there are two LUTs.
} 
placed at at least one of the sites $A$ and $B$. The two equations above guarantee that $X$ will be placed at exactly one location. Similar constraints would be generated for the flip-flop $Z$ and the possible locations $D$ and $E$.

Nets are treated like logic element sites, which are encoded as Boolean variables, $U_{i} @ N_{j}(\forall i \in\{1 \cdots 8\}, j \in\{0 \cdots 15\})$. The mutual exclusion conditions apply to nets as well, so that distinct nets in the user netlist cannot be present at any one net in the PLB.

We also have input and output constraints for all logic elements. One such output constraint would be

$$
X @ A \rightarrow U_{5} @ N_{10},
$$

which guarantees that if $X$ is placed at location $A$ in the PLB, then the $N_{10}$ net in the PLB carries the $U_{5}$ signal. Input constraints are also be generated in an analogous fashion.

For routing multiplexers (like $G$ and $H$ ) which are controlled by configuration logic we have variables that determine which input drives the output. So for $G$, we would have the variables, $G_{0 \rightarrow \text { out }}$ and $G_{1 \rightarrow \text { out }}$, and we need to add exclusive driver constraints (19) so that no output is driven by two inputs. We also need to add routing constraints (20) so that if a net is present at an input to a routing multiplexer and the appropriate control bit is active, the output has the correct net.

$$
\begin{array}{r}
G_{0 \rightarrow \text { out }} \rightarrow\left(\neg G_{1 \rightarrow \text { out }}\right) \\
\left(G_{0 \rightarrow \text { out }} \wedge U_{5} @ N_{10}\right) \rightarrow U_{5} @ N_{12}
\end{array}
$$

Finally, extra variables are needed to encode the choice that some logic elements can act as route-throughs. An example of such an element is a lookup table. It can be programmed to pass one of its inputs out to the output and thus act like a routing multiplexer if it is not being used. This increases the routing flexibility if we need it. Corresponding to these extra variables, route-through constraints are generated. For example, some of the constraints generated for taking into consideration that LUT $B$ might be used as a route-through are

$$
\begin{array}{r}
\left(R T_{B} \wedge B_{0 \rightarrow \text { out }}\right) \rightarrow\left(\neg B_{1 \rightarrow \text { out }}\right) \\
\left(R T_{B} \wedge B_{0 \rightarrow \text { out }} \wedge U_{8} @ N_{5}\right) \rightarrow U_{8} @ N_{11}
\end{array}
$$

where $R T_{B}$ encodes the choice as to whether LUT $B$ is used as a route-through. Constraint (21) maintains that inputs 0 and 1 cannot drive the output simultaneously. There will be other constraints corresponding to mutual exclusion of every pair of inputs. Constraint (22) specifies that if input 0 does drive the output and if net $N_{5}$ is at input 0 , then it would also appear at the output. Note that constraint (21) is just a representative constraint and other constraints related to the other input pins and to other possible nets will also be generated as needed.

Certain input pins of logic elements are swappable, i.e., they may be interchanged if doing so helps in producing a valid PLB. Variables are introduced to encode the choice of equivalent pins that have been swapped and equivalent pin constraints corresponding to these are added.

Finally, boundary constraints forcing input (or output) nets to be assigned to at least one of the input (or output) pins are added. An example of such a constraint is

$$
U_{6} @ N_{13} \vee U_{6} @ N_{14} \vee U_{6} @ N_{15}
$$

Once all the constraints are automatically generated from a topological description of the PLB graph and the logic elements in the user's netlist that we wish to pack into a PLB, we use a SAT-solver to check if they can all be satisfied simultaneously. If they are all satisfied, we can generate a valid placement and route from the solution that the SAT solver gives us. Translating the solution to an assignment of netlist elements to PLB locations, PLB input pins to nets and control bits in the routing multiplexers is easy. We construct the PLB based on all the variables that are TRUE in the satisfying assignment. In our example, one satisfying assignment would have the following variables be TRUE: $U_{1} @ N_{0}, U_{2} @ N_{1}, \cdots, X @ A, R T_{B}$, where $U_{1} @ N_{0}$ means that the net $U_{1}$ is at the PLB input $N_{0}, X @ A$ means that LUT $X$ is placed at location $A$ and $R T_{B}$ means that LUT $B$ is used as a route-through.

\section{ARCHITECTURE EVALUATION}

\subsection{Area and Delay Modeling}

Suggested in [3], a LUT-4 can be implemented by 161-bit SRAM, 4 inverters (INV1) and 15 2:1MUXes which are $16 \times 6+4 \times 2+$ $15 \times 4=164$ transistors, and a LUT- 6 can be implemented by 64 1-bit SRM, 6 inverters and 63 2:1MUXes, which are $64 \times 6+$ $6 \times 2+63 \times 4=648$ transistors. The macro-gate in Figure 3 can be implemented by 9 1-bit SRM, 7 inverters, 3 2:1MUXes and 84 transistors (for implementing the macro-gate ${ }^{5}$ ), which are $9 \times 6+7 \times 2+3 \times 4+84=164$ transistors. The logic delay is modeled by the square root of the logic area. The area and delay models used in our experiments are summarized in Table 2.

\begin{tabular}{|c|c|c|c|}
\hline & LUT-4 & macro-gate & LUT-6 \\
\hline transistor number & 164 & 164 & 648 \\
normalized area & 1.00 & 1.00 & 3.95 \\
normalized delay & 1.00 & 1.00 & 1.98 \\
\hline
\end{tabular}

Table 2: Summary of the area and delay models

\subsection{Experimental Results}

We implement the improved mapping algorithms in the Berkeley ABC platform [8] with C language and the SAT-based packer with LISP language, and test them on IWLS'05 benchmarks [7]. The MBILP and SAT problem are solved by mosek [24] and miniSAT [25], respectively.

In our technology mapping, the combinational portion of an application is first mapped to LUTs and macro-gates, and the sequential elements are added back to the mapped sub-circuit. The mapped application is then packed by our SAT-based packer. After the packing, the logic cost is evaluated based on the aforementioned area and delay model. Note that the placement and routing are not performed because this work is focused on logic delay and area evaluation for the proposed macro-gate based heterogeneous FPGA architecture. The interconnect cost of such architecture is not considered here and will instead be evaluated in our future work.

Table 1 compares four architectures, i.e., a PLB containing a LUT-4 (column "LUT4"), the mix of a LUT-4 and a macro-gate in Figure 3 (column "LUT4+MG"), a LUT-6 (column "LUT6"), and the mix of a LUT-6 and a macro-gate (column "LUT6+MG"), respectively. The logic depth, number of PLBs, estimated delay and area $^{6}$ of the circuits with different architectures are shown in the table. Compared to LUT-4 only architecture, the mix of LUT4 and macro-gates reduces both logic depth and logic delay by $9.2 \%$, and reduces logic area by $12.9 \%$. Compared to LUT-6 only architecture, the mix of LUT- 6 and macro-gates reduces logic delay and logic area by $16.5 \%$ and $30 \%$, respectively, while it increases

\footnotetext{
${ }^{5}$ The embedded logic gates $g_{1} \cdots g_{4}$ of the macro-gate in Figure 3 are synthesized by SIS 1.2 [22] with batch command script.rugged followed by read_library mcnc.genlib; map - $m 0$, and their schematics are omitted due to the space limit

${ }^{6}$ Given areas in Table 2, the area ratio between these four PLBs is 1.00: $(1.00+1.00): 3.98:(3.98+1.00)$.
} 


\begin{tabular}{|c|c|c|c|c|c|c|c|c|c|c|c|c|c|c|c|c|}
\hline circuit & \multicolumn{4}{|c|}{ Logic depth } & \multicolumn{4}{|c|}{ Estimated delay (normalized) } & \multicolumn{4}{|c|}{ PLB\# } & \multicolumn{4}{|c|}{ Estimated area (normalized) } \\
\hline & LUT4 & \begin{tabular}{|c|} 
LUT4 \\
MG
\end{tabular} & LUT6 & \begin{tabular}{|c|} 
LUT6 \\
MG
\end{tabular} & LUT4 & $\begin{array}{c}\text { LUT4 } \\
\text { MG }\end{array}$ & LUT6 & $\begin{array}{c}\text { LUT6 } \\
\text { MG }\end{array}$ & LUT4 & $\begin{array}{c}\text { LUT4 } \\
\text { MG }\end{array}$ & LUT6 & $\begin{array}{c}\text { LUT6 } \\
\text { MG }\end{array}$ & LUT4 & $\begin{array}{c}\text { LUT4 } \\
\text { MG }\end{array}$ & LUT6 & \begin{tabular}{|c|} 
LUT6 \\
MG
\end{tabular} \\
\hline ac97_ctrl & 4 & 4 & 3 & 4 & 4 & 4 & 6 & 4 & 4010 & 1739 & 2898 & 1523 & 4599 & 3733 & 13132 & 8424 \\
\hline aes_core & 8 & 7 & 6 & 9 & 8 & 7 & 12 & 10 & 8959 & 4147 & 3912 & 2932 & 10275 & 8903 & 17727 & 16218 \\
\hline des_area & 10 & 10 & 7 & 10 & 10 & 10 & 14 & 11 & 2153 & 1004 & 968 & 674 & 2469 & 2154 & 4386 & 3728 \\
\hline des_perf & 6 & 6 & 4 & 7 & 6 & 6 & 8 & 7 & 32371 & 15652 & 6872 & 4863 & 37125 & 33601 & 31140 & 26900 \\
\hline ethernet & 10 & 9 & 7 & 9 & 10 & 9 & 14 & 12 & 5047 & 2438 & 3953 & 2215 & 5788 & 5234 & 17913 & 12252 \\
\hline $\mathrm{i} 2 \mathrm{c}$ & 5 & 4 & 4 & 4 & 5 & 4 & 8 & 6 & 392 & 188 & 265 & 144 & 450 & 404 & 1201 & 794 \\
\hline mem_ctrl & 11 & 9 & 8 & 9 & 11 & 9 & 16 & 12 & 3687 & 1731 & 2892 & 1413 & 4228 & 3715 & 13105 & 7813 \\
\hline pci_bridge32 & 9 & 8 & 7 & 8 & 9 & 8 & 14 & 11 & 6596 & 3156 & 5413 & 2911 & 7565 & 6774 & 24529 & 16102 \\
\hline pci_spoci_ctrl & 6 & 5 & 5 & 5 & 6 & 5 & 10 & 7 & 372 & 173 & 251 & 137 & 427 & 370 & 1137 & 755 \\
\hline sasc & 3 & 3 & 2 & 4 & 3 & 3 & 4 & 4 & 206 & 87 & 147 & 85 & 236 & 186 & 666 & 470 \\
\hline simple_spi & 4 & 4 & 3 & 4 & 4 & 4 & 6 & 4 & 295 & 121 & 200 & 113 & 338 & 259 & 906 & 625 \\
\hline spi & 10 & 9 & 7 & 9 & 10 & 9 & 14 & 11 & 1315 & 601 & 913 & 440 & 1508 & 1289 & 4137 & 2431 \\
\hline ss_pcm & 3 & 3 & 2 & 3 & 3 & 3 & 4 & 3 & 124 & 61 & 102 & 48 & 142 & 131 & 462 & 266 \\
\hline systemcaes & 11 & 11 & 8 & 13 & 11 & 11 & 16 & 16 & 2948 & 1470 & 1926 & 1158 & 3381 & 3155 & 8728 & 6405 \\
\hline systemcdes & 9 & 8 & 5 & 9 & 9 & 8 & 10 & 10 & 1125 & 580 & 608 & 351 & 1290 & 1245 & 2755 & 1942 \\
\hline $\operatorname{tv} 80$ & 17 & 15 & 11 & 15 & 17 & 15 & 22 & 20 & 2963 & 1404 & 2132 & 1129 & 3398 & 3013 & 9661 & 6245 \\
\hline usb_funct & 9 & 9 & 6 & 9 & 9 & 9 & 12 & 10 & 4906 & 2227 & 3408 & 1775 & 5626 & 4781 & 15443 & 9816 \\
\hline usb_phy & 4 & 3 & 2 & 3 & 4 & 3 & 4 & 4 & 188 & 89 & 145 & 60 & 216 & 190 & 657 & 332 \\
\hline vga_lcd & 7 & 7 & 6 & 7 & 7 & 7 & 12 & 9 & 40814 & 18145 & 28791 & 16561 & 46808 & 38954 & 130466 & 91604 \\
\hline wb_conmax & 9 & 7 & 6 & 7 & 9 & 7 & 12 & 10 & 14580 & 6966 & 11003 & 5895 & 16721 & 14954 & 49860 & 32608 \\
\hline wb_dma & 10 & 9 & 6 & 9 & 10 & 9 & 12 & 11 & 1469 & 711 & 1129 & 579 & 1685 & 1525 & 5116 & 3200 \\
\hline average & 7.86 & 7.14 & 5.48 & 7.48 & 7.86 & 7.14 & 10.95 & 9.14 & 6406 & 2985 & 3711 & 2142 & 7346 & 6408 & 16816 & 11849 \\
\hline
\end{tabular}

Table 1: Delay and area comparisons among different architectures

the logic depth by $36.5 \%$. The average logic depth of mixing LUT-6 and macro-gate is larger than that for mixing LUT-4 macro-gates because our technology mapper sets the delay weight of a LUT-6 and a macro-gate as 1.98:1.00 based on Table 2. The experimental results show that the logic area is reduced by $5 \%$ due to the MBILP based area recovery algorithm (Subsection 3).

\section{CONCLUSIONS AND FUTURE WORK}

Targeting macro-gate based heterogeneous FPGAs, a methodology has been proposed to extract a small set of logic functions that are able to implement a large portion of functions for given FPGA applications. Assuming that the extracted logic functions are implemented by macro-gates in PLBs, a complete synthesis flow has been developed for such heterogeneous PLBs with mixed LUTs and macro-gates. The flow includes a cut-based delay-optimal technology mapping, a mixed binary integer and linear programming based area recovery algorithm to balance the resource utilization of macrogates and LUTs for area-efficient packing, and a SAT-based packing. The proposed heterogeneous FPGA design has been evaluated using the newly developed flow. The experimental results show that mixing LUT and macro-gates, both with 6 inputs, improves performance by $16.5 \%$ and reduces logic area by $30 \%$ compared to using 6-input LUTs.

In the future, we will investigate more complicated macro-gate based heterogeneous PLBs architectures by the proposed synthesis flow, and consider area, delay and power optimization during the technology mapping and packing processes. We will also connect our synthesis flow with the back-end tools such as placement and routing for even more accurate architecture evaluation considering both logic and routing cost.

\section{REFERENCES}

[1] V. Betz, J. Rose, and A. Marquardt, Architecture and CAD for Deep-Submicron FPGAs. Kluwer Academic Publishers, 1999.

[2] "The programmable gate array data book," Xilinx Inc., 1989.

[3] J. Cong, et al, "Technology mapping and architecture evaluation for k/m-macrocell-based FPGAs," TOADES, 2005.

[4] "Xilinx datasheets," http://www.xilinx.com/literature.

[5] A. Cosoroaba and F. Rivoallon, "Achieving higher system performance with the virtex-5 family of FPGAs," in http://www.xilinx.com/literature.

[6] F. Li and L. He, "Power modeling and characteristics of field programmable gate arrays," TCAD, 2005.
[7] http://iwls.org/iwls2005/benchmarks.html.

[8] http://www.eecs.berkeley.edu/alanmi/abc/.

[9] J. Cong and S. Xu, "Delay-optimal technology mapping for FPGAs with heterogeneous LUTs," $D A C, 1998$.

[10] J. Cong, et al, "Cut ranking and pruning: Enabling a general and efficient fpga mapping solution," FPGA, 1999.

[11] A. Kaviani and S. Brown, "Hybrid FPGA architecture," FPGA, 1996.

[12] J. Cong and Y. Ding, "Flowmap: An optimal technology mapping algorithm for delay optimization in lookup-table based FPGA designs," TCAD, 1994.

[13] J. Cong and Y. Ding, "On area/depth trade-off in LUT-based FPGA technology mapping," TVLSI, 1994.

[14] D. Chen and J. Cong, "Daomap: A depth-optimal area optimization mapping algorithm for FPGA designs," ICCAD, 2004.

[15] V. Manohara-rajah, et al, "Heuristics for area minimization in LUT-based FPGA technology mapping," IWLS, 2004.

[16] A. Mishchenko, et al, "Integrating logic synthesis, technology mapping, and retiming," IWLS, 2005.

[17] S. Chatterjee, et al, "Factor cuts," ICCAD, 2006.

[18] A. Ling, et al, "Fpga logic synthesis using quantified boolean satisfiability," SAT 2005, pp. 444-450.

[19] S. Safarpour, et al, "Efficient satbased boolean matching for fpga technology mapping," $D A C, 2006$.

[20] D. Chai and A. Kuehlmann, "Building a better boolean matcher and symmetry detector," DATE, 2006.

[21] S. C. Soheil Ghiasi, et al, "A unified theory of timing budget management," $D A C 2004$.

[22] E. M. Sentovich, et al, "SIS: A system for sequential circuit systhesis," Department of Electrical Engineering and Computer Science, Berkeley, CA 94720, 1992.

[23] J. M. Rabaey, et al, Digital Integrated Circuits: A Design Perspective (Second Edition). Prentice-Hall, 2003.

[24] http://www.mosek.com.

[25] http://www.cs.chalmers.se/Cs/Research/ FormalMethods/MiniSat/MiniSat.html.

[26] Jianshe He, "Technology Mapping and Architecture of Heterogenous Field-Programmable Gate Arrays," M.A.Sc. Thesis, University of Toronto, 1993.

[27] Kevin Chung, "Architecture and Synthesis of Field-Programmable Gate Arrays with Hard-wired Connections," Ph.D. Thesis, University of Toronto, 1994. 